\title{
Ventricular Tachycardia and Sudden Cardiac Death
}

\author{
Bruce A. Koplan, MD, MPH, and William G. Stevenson, MD
}

Ventricular tachycardia (VT), which most commonly occurs in patients with structural heart disease, can be associated with an increased risk of sudden death. The most common cause of ventricular fibrillation is acute coronary ischemia, whereas a myocardial scar from prior infarct is the most common cause of sustained monomorphic VT in patients with structural heart disease. More benign forms of idiopathic VT can also occur in the absence of structural heart disease. Treatment of VT involves both emergent management and prevention of recurrence with medical and device therapy. Appropriately selected patients who have experienced VT or those who are at risk of VT may be candidates for an implantable cardioverter-defibrillator. The left ventricular ejection fraction is most frequently used to stratify patients with either ischemic or nonischemic cardiomyopathy who are at risk of sudden death and may be candidates for a prophylactic defibrillator. Catheter ablation may also be an option for appropriately selected patients with many forms of VT. This article discusses the etiologies and management of VT and its association with sudden death.

Mayo Clin Proc. 2009;84(3):289-297

ARVD = arrhythmogenic right ventricular dysplasia; ECG = electrocardiogram; ICD = implantable cardioverter-defibrillator; $L V E F=$ left ventricular ejection fraction; $\mathrm{MI}=$ myocardial infarction; RVOT $=$ right ventricular outflow tract; $\mathbf{V F}=$ ventricular fibrillation; VT = ventricular tachycardia

$\mathrm{V}$ entricular tachycardia (VT) can be classified according to duration, morphology, and hemodynamic effect. Nonsustained VT terminates spontaneously without substantial hemodynamic compromise. Sustained VT is VT that lasts longer than 30 seconds and/or requires an intervention for termination or produces severe hemodynamic compromise or syncope before terminating spontaneously. Ventricular tachycardia is described as monomorphic when each QRS complex resembles the next. When QRS complexes during tachycardia vary in appearance from beat to beat, VT is classified as polymorphic (Figure 1). Torsades de pointes is a form of polymorphic VT that is often associated with a prolonged QT interval.

\section{ETIOLOGIES}

Ventricular Tachycardia With Structural Heart Disease Although usually associated with structural heart disease, VT can occur in its absence. Ischemic heart disease is the most common cause of sustained ventricular arrhythmias. Acute coronary ischemia is a cause of polymorphic VT or ventricular fibrillation (VF) and is probably the most common cause of out-of-hospital sudden death. During acute ischemia, the leakage of potassium leads to increased extracellular potassium that depolarizes myocytes in the ischemic border zone. ${ }^{1,2}$ This depolarization leads to electrical heterogeneity of conduction and refractoriness that provide a substrate for reentry, resulting in polymorphic VT and/or VF.

Sustained monomorphic VT that is due to structural heart disease is most commonly a result of reentry involving a region of myocardial scar. The most common cause of a scar is an old infarct. This type of VT occurs in the absence of acute ischemia. ${ }^{3,4}$ Ventricular scars leading to reentrant VT also occur in nonischemic cardiomyopathies, including idiopathic dilated cardiomyopathy, hypertrophic cardiomyopathy, infiltrative heart disease (eg, sarcoidosis), right ventricular dysplasia, and after repair of congenital heart disease or valvular heart disease.

Bundle branch reentry is a unique form of reentrant monomorphic VT that does not require a myocardial scar. It involves a reentrant circuit that uses the specialized conduction system with the circulating wavefront traveling antegrade down the right bundle branch and retrograde up the left bundle branch, leading to a typical left bundle branch block morphology during VT. Less commonly, the circuit may travel in the opposite direction, or it may use the left anterior and posterior fascicles only, causing VT with a right bundle branch block configuration. This VT is associated with severe conduction disease; most patients have intraventricular conduction defects on their baseline electrocardiogram (ECG) and ventricular dysfunction that is often severe. Bundle branch reentry accounts for approximately $5 \%$ to $8 \%$ of all sustained monomorphic VTs in patients referred for catheter ablation. ${ }^{5,6}$ Although it is relatively uncommon, recognition of this arrhythmia is important because it is amenable to catheter ablation therapy. ${ }^{7,8}$

\section{Familial Causes of VT and VF}

A number of genetic conditions cause polymorphic ventricular arrhythmias and sudden death in the absence of

From the Cardiac Arrhythmia Section, Brigham and Women's Hospital, Harvard Medical School, Boston, MA.

Address correspondence to Bruce A. Koplan, MD, MPH, Cardiac Arrhythmia Section, Brigham and Women's Hospital, 75 Francis St, Boston, MA 02446 (bkoplan@partners.org). Individual reprints of this article and a bound reprint of the entire Symposium on Cardiovascular Diseases will be available for purchase from our Web site www.mayoclinicproceedings.com.

(C) 2009 Mayo Foundation for Medical Education and Research 


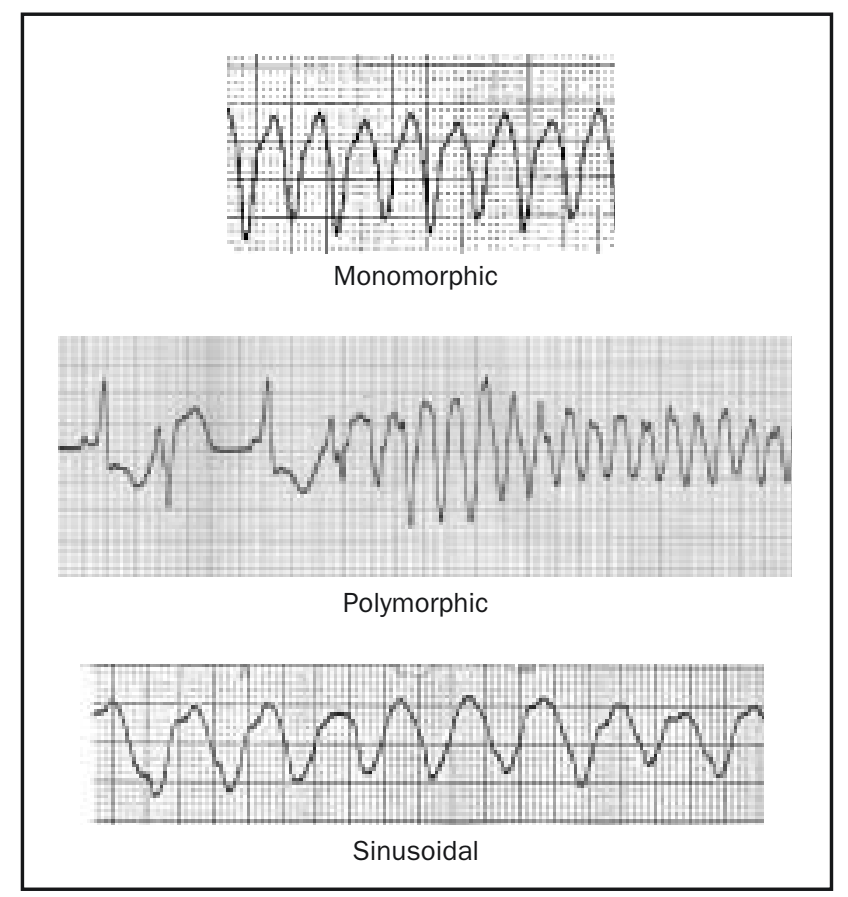

FIGURE 1. Electrograms showing 3 different types of ventricular tachycardia.

visible structural heart disease (Table 1). Most of these disorders are ion channel abnormalities. Long QT syndrome results most often from abnormalities of either potassium or sodium ion channels and typically manifests as a prolonged QT interval on the surface ECG. ${ }^{9}$ Syncope and sudden death result from polymorphic VT that often has waxing and waning QRS amplitude known as torsades de pointes.

In patients with Brugada syndrome, incomplete right bundle branch block and ST-segment elevation in the anterior precordial leads is evident on ECG. ${ }^{10} \mathrm{~A}$ genetic defect causing abnormal sodium channel function causes approximately a quarter of cases. Syncope and sudden death result from polymorphic VT, tend to occur during sleep, and can be precipitated by fever. Findings on ECG, which can wax and wane over time, can be exacerbated by administration of sodium channel-blocking agents, such as flecainide.

Catecholaminergic polymorphic VT is an inherited disorder in which abnormal calcium processing causes exer-

\section{TABLE 1. Inherited Disorders Associated With Sudden Death}

Long QT syndrome

Short QT syndrome

Hypertrophic cardiomyopathy

Familial dilated cardiomyopathy

Brugada syndrome

Catecholaminergic polymorphic ventricular tachycardia

Arrhythmogenic right ventricular dysplasia cise-induced polymorphic VT. ${ }^{11}$ Most patients present during childhood with syncope or cardiac arrest. Sequence variations in the cardiac ryanodine receptor or calsequestrin are identified causes. ${ }^{12,13}$

Arrhythmogenic right ventricular dysplasia (ARVD) is an inherited disorder that may present with monomorphic VT or polymorphic VT. It is associated with fibrofatty replacement of the right ventricle, leading to abnormal right ventricular function and abnormal structural appearance on cardiac imaging; however, the involvement can be patchy and difficult to detect. ${ }^{14,15}$ Sequence variations in genes coding for proteins involved in cell-to-cell adhesions in the desmosome are the most often identified cause. The electrogram in patients with ARVD may show prolonged QRS duration, incomplete or complete right bundle branch block, right precordial T-wave inversions, and/or a distinct wave just beyond the QRS complex referred to as an epsilon wave.$^{16}$ Monomorphic VT typically has a left bundle branch block configuration due to its origin in the right ventricle.

In view of these recognized genetic cause of syndromes causing VT or VF, a family history of sudden death is often an important finding that mandates careful evaluation for these conditions in patients presenting with ventricular arrhythmias.

\section{IDIOPATHIC VT}

Ventricular tachycardia that occurs in the absence of structural heart disease, genetic conditions such as long QT syndrome, or metabolic/electrolyte abnormalities is referred to as idiopathic VT. Idiopathic VTs tend to originate from a few specific locations that can be recognized from the ECG. Most idiopathic VTs originate from the right ventricular outflow tract (RVOT) and thus most commonly have a left bundle branch block morphology and an inferiorly directed axis. ${ }^{17,18}$ Idiopathic RVOT tachycardia originates from a focus, with automaticity mediated by cyclic adenosine monophosphate-dependent triggered activity leading to intracellular calcium overload. ${ }^{19,20}$ Although most originate from the right side of the outflow tract region, some originate from the left ventricular outflow tract. These VTs may be sustained or may present as repetitive runs of nonsustained VTs, referred to as repetitive monomorphic VTs. ${ }^{21}$

A less common form of idiopathic VT results from reentry involving the fascicles of the left bundle branch, leading to VT with a right bundle branch block configuration, usually with a superiorly directed QRS-axis tachycardia. Interestingly, this tachycardia is sensitive to verapamil..$^{22}$ Idiopathic forms of VT are rarely life-threatening but may be associated with hemodynamic compromise and syncope when rapid and sustained. ${ }^{23}$ 


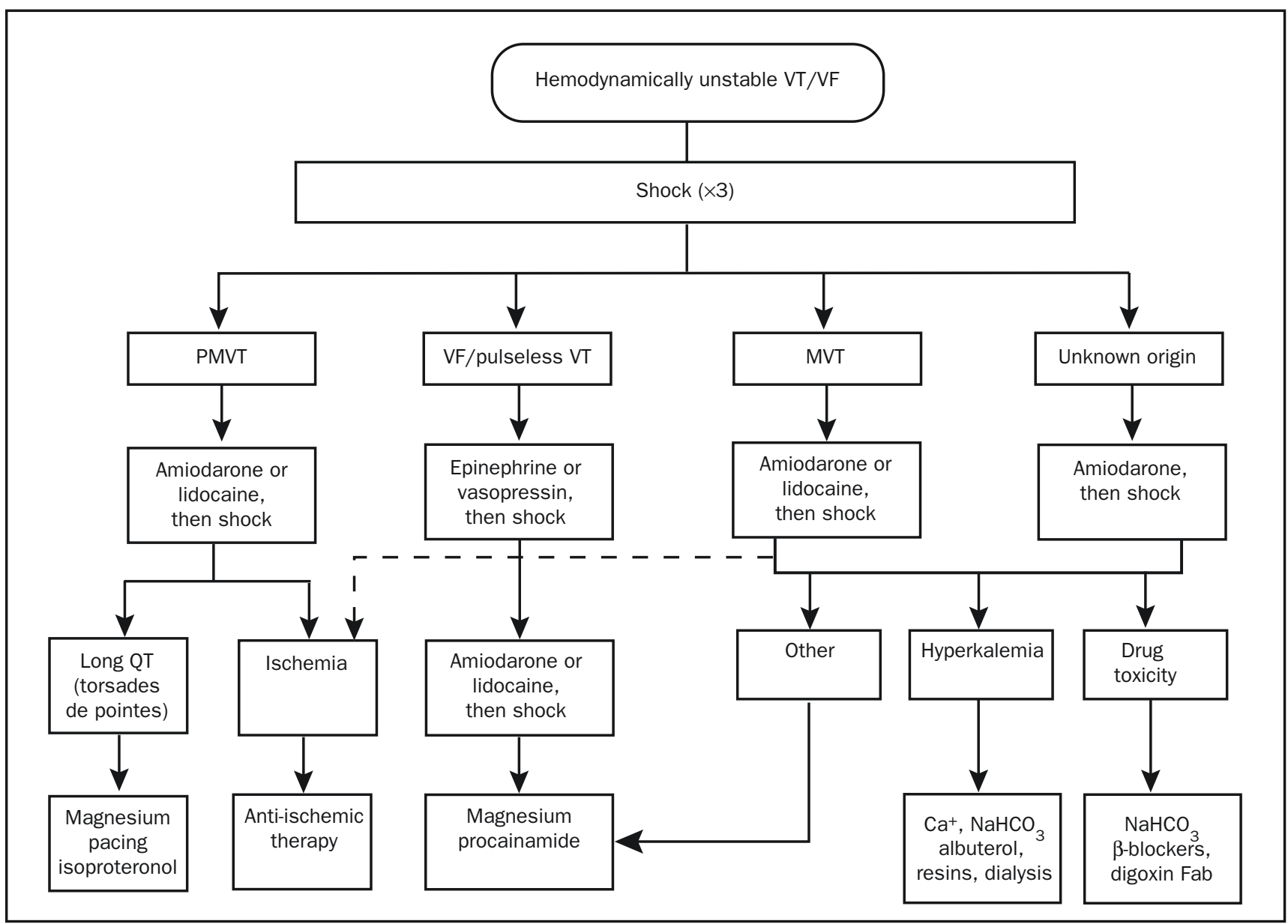

FIGURE 2. Emergent management of hemodynamically unstable ventricular tachycardia (VT)/ventricular fibrillation (VF). Fab $=$ fragment antigen binding compound; $\mathrm{MVT}=$ monomorphic VT; $\mathrm{NaHCO}_{3}=$ bicarbonate of soda; PMVT= polymorphic VT. Adapted from the American Heart Association Guidelines for Cardiopulmonary Resuscitation and Emergency Cardiovascular Care. ${ }^{24}$

\section{MANAGEMENT OF VT}

\section{Emergent Management and Initial Evaluation}

Regardless of the etiology, emergent cardioversion is warranted for sustained VT that is causing symptomatic hypotension, pulmonary edema, or myocardial ischemia. An algorithm for the emergent resuscitation of ventricular arrhythmias (adapted from The American Heart Association Guidelines for Cardiopulmonary Resuscitation and Emergency Cardiovascular Care) is shown in Figure 2. ${ }^{24}$ Reversible causes, such as acute ischemia, electrolyte abnormalities, or drug toxicities, should be corrected.

Premature ventricular beats and nonsustained VT can be an indication of underlying heart disease, myocardial ischemia, hypoxia, or electrolyte abnormalities. Evaluation for these potential provocative factors is warranted. These arrhythmias are common in patients with depressed ven- tricular function; their frequency increases with the severity of left ventricular dysfunction. Although ventricular ectopy and nonsustained VT are markers for increased mortality, pharmacological suppression of these arrhythmias does not reduce sudden death.

After stabilization, most patients warrant an assessment of structural heart disease with ECG or other imaging technique (eg, cardiac magnetic resonance imaging). Coronary artery disease should be excluded when this is a possibility. Further therapy is guided by the nature of underlying heart disease and the specific type of ventricular arrhythmia. Polymorphic VT suggests myocardial ischemia or an inherited arrhythmogenic syndrome. Sustained monomorphic VT may be idiopathic, occurring in the absence of structural heart disease and associated with a benign prognosis; however, it more commonly indicates the presence of a myocardial scar that provides the sub- 
strate for reentry. The prognosis (particularly the risk of sudden death) and the therapy required are determined largely by the underlying heart disease.

\section{Sudden Death and Ventricular Arrhythmias}

Sudden death accounts for greater than half of all cardiac deaths and up to $15 \%$ of total mortality in the United States. ${ }^{25}$ In epidemiological studies, sudden death is typically defined as unexpected death that occurs immediately or within 1 hour of an abrupt change from a stable clinical state. Sudden death is frequently due to VT degenerating to VF. Numerous risk factors for sudden cardiac death have been identified. It is more than twice as likely in men as in women and more then 3 to 5 times as likely in the setting of structural heart disease, particularly coronary artery disease. ${ }^{26,27}$ Greater than two-thirds of sudden cardiac arrests are associated with coronary artery disease. It can be the first manifestation of coronary artery disease, making detection of high-risk patients problematic.

Patients who survive a myocardial infarction (MI) are at risk, with the incidence of sudden death or cardiac arrest being highest ( $1.40 \%$ per month) in the first month after MI and decreasing to $0.14 \%$ per month at 2 years after MI. ${ }^{28}$ One of the most easily identified and most commonly used noninvasive risk factors for sudden death is a depressed left ventricular ejection fraction (LVEF). ${ }^{29}$ An LVEF of $30 \%$ or less is associated with an annual overall mortality rate approaching $10 \% .^{30,31}$ Other risk factors for sudden death include abnormal heart rate variability, microvolt T-wave alternans, and ambient ventricular arrhythmias. ${ }^{32-35} \mathrm{Al}-$ though these noninvasive risk factors have been shown to be associated with an increased incidence of sudden cardiac death in selected patient populations, their usefulness in selecting which patients would benefit from an implantable cardioverter-defibrillator (ICD) remains questionable and is still under study. Inducible VT during invasive electrophysiological study with programmed ventricular stimulation is also a risk factor for sudden cardiac death in patients with ischemic heart disease, for which an ICD is usually warranted. ${ }^{36}$

Patients with depressed ventricular function from cardiomyopathies, congenital heart disease, and valvular heart disease are also at risk of VT and sudden death. Risk factors are less well defined in these populations than for populations with coronary artery disease. Although development of large areas of ventricular scar is not common in nonischemic cardiomyopathies, sustained VT often seems to be related to a scar that can be identified as areas of delayed gadolinium enhancement on cardiac magnetic resonance imaging. Whether magnetic resonance imaging will be helpful in identifying patients at risk is an area of active interest and is under investigation. ${ }^{37}$
A small but important fraction of sudden deaths are due to genetic causes of cardiomyopathy (hypertrophic or dilated) or to ion channel abnormalities (eg, long QT syndrome, short QT syndrome, Brugada syndrome). A family history of sudden death should prompt careful consideration for these entities. Interestingly, a familial predisposition to sudden death in patients at risk of coronary artery disease has also been shown; a genetic susceptibility to VF during ischemia is one of the hypotheses invoked to explain this observation.

\section{Pharmacological Therapy for Ventricular Arrhythmias}

$\beta$-Blockers and angiotensin-converting enzyme inhibitors have been shown to reduce mortality and sudden death in patients with coronary artery disease or heart failure. ${ }^{38-40}$ Ventricular tachycardia is often sensitive to adrenergic stimulation, and $\beta$-blockers can reduce arrhythmia recurrences. Membrane-active antiarrhythmic drugs have largely been a disappointment and do not reduce mortality in patients at risk of ventricular arrhythmias. ${ }^{41,42}$ Class III antiarrhythmic drugs (eg, sotalol and amiodarone) have been shown to reduce arrhythmia recurrences but not mortality. ${ }^{43,44}$ Class I antiarrhythmic drugs (eg, quinidine, flecainide, and propafenone) actually increase mortality in patients with ventricular arrhythmias and prior MI. ${ }^{41,45,46}$ In patients with structural heart disease and sustained VT or VF, antiarrhythmic drugs may be used to reduce recurrent symptomatic arrhythmias after placement of an ICD.

Idiopathic VT often responds to $\beta$-blockers or the nondihydropyridine calcium channel-blocking agents (verapamil or diltiazem). Class I or III antiarrhythmics can also be effective, but catheter ablation is usually considered if $\beta$ blockers and calcium channel blockers are not effective, rather than long-term antiarrhythmic medications. Catheter ablation of idiopathic VT can be successfully performed in approximately $80 \%$ of patients. ${ }^{47}$

\section{Role of ICDs in Patients at Risk of Sudden Death}

Implantable cardioverter-defibrillators effectively terminate VT and VF for most patients and reduce mortality in selected patients at risk of sudden death. Appropriate selection of patients for ICD therapy has been the subject of extensive investigation. Two broad categories of patients are candidates for ICD therapy: those receiving an ICD for secondary prevention of sudden death after surviving an episode of cardiac arrest and those treated for primary prevention of sudden death who have not had a prior cardiac arrest or sustained VT.

Patients who have survived a cardiac arrest or who have sustained VT that causes hemodynamic compromise and is not due to a secondary cause (such as acute MI) have a high risk $(>40 \%)$ of having a recurrent episode of VT or VF in the 


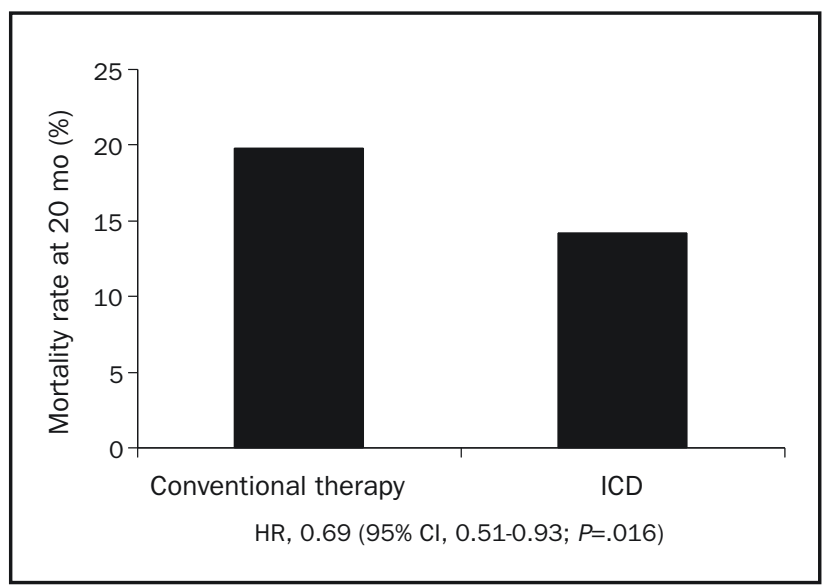

FIGURE 3. Twenty-month mortality rate in patients with ischemic heart disease and left ventricular ejection fraction of $\leq 30 \%$ from the second Multicenter Automatic Defibrillator Implantation Trial (MADIT-II). Cl = confidence interval; $\mathrm{HR}=$ hazard ratio; ICD = implantable cardioverterdefibrillator.

next 2 years. An ICD for secondary prevention improves survival ( $31 \%$ reduction in mortality in 3 years). ${ }^{28}$

Implantable cardioverter-defibrillators also reduce mortality rates in selected high-risk patients for primary prevention. Sudden death risk is best identified from depressed left ventricular function. In the second Multicenter Automatic Defibrillator Implantation Trial (MADIT II) (Figure 3), ICDs for patients with coronary artery disease and an LVEF of $30 \%$ or less reduced mortality by $5.6 \%$ at 20 months (relative risk reduction of $28 \%$ ) compared with conventional medical treatment alone..$^{30}$ The Sudden Death in Heart Failure Trial (SCD-HeFT) enrolled patients with an LVEF of $35 \%$ or less due to either ischemic or nonischemic heart disease who had New York Heart Association class II or III heart failure. ${ }^{48}$ At 5 years of follow-up, ICDs reduced mortality compared with amiodarone $(28.9 \%$ vs $34.1 \%$ ) or standard medical therapy (28.9\% vs 35.8\%) (Figure 4). Amiodarone was of no benefit compared with standard medical therapy. ${ }^{48}$ Meta-analyses further support a survival benefit for ICD therapy in patients with nonischemic cardiomyopathy. ${ }^{49}$

However, selection for ICD therapy must also be individualized. Patients with serious comorbidities associated with a survival of less than 1 year should generally not be considered candidates for ICD therapy; they are unlikely to benefit and would be subject to potential adverse effects of the ICD. Furthermore, although advanced age is not an absolute contraindication to device therapy, comorbidities can have profound effects on expected mortality in elderly patients, and decisions to implant ICDs must be individualized in this group. ${ }^{50}$

Table 2 summarizes some of the more common approved indications for ICD therapy for either primary or

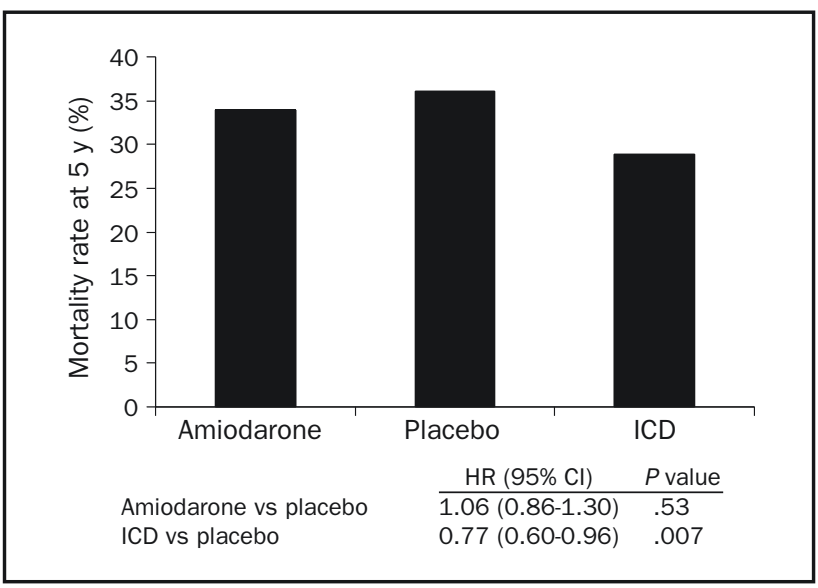

FIGURE 4. Five-year mortality rate in patients with cardiomyopathy of either ischemic or nonischemic etiology from the Sudden Cardiac Death in Heart Failure Trial (SCD-HeFT). Cl = confidence interval; HR = hazard ratio; ICD = implantable cardioverter-defibrillator.

secondary prevention of sudden death adapted from the Centers for Medicare and Medicaid Services 2005 guidelines. Primary prevention indications for ICD therapy are based mainly on the measured LVEF; ICD therapy is indicated only if the LVEF has been reduced for an extended period and is not likely to recover. Reassessment of ventricular function is recommended several weeks after acute

\section{TABLE 2. Indications and Contraindications for ICD Therapy ${ }^{a}$}

Indications
Primary prevention of sudden death
Ischemic heart disease
IDCM, documented prior MI, at least 40 d status post-MI,
NYHA class II and III, and measured LVEF $\leq 35 \%$
Ischemic heart disease, LVEF $\leq 40 \%$, and inducible VT on
electrophysiologic study
Nonischemic heart disease
NIDCM >9 mo, NYHA class II and III, LVEF $\leq 35 \%$
Familial or inherited conditions with high risk of sudden death
Diseases associated with a substantial risk of sudden death
Congenital syndromes: long QT interval, Brugada syndrome,
ARVD, hypertrophic cardiomyopathy with high-risk features
Secondary prevention of sudden death
Documented cardiac arrest due to VF or hemodynamically
unstable VT, not due to a transient or reversible cause
Contraindications
CABG or PTCA within 3 mo or MI within $40 \mathrm{~d}$
Candidacy for revascularization
Irreversible brain damage
Any other disease associated with a likelihood of survival of <1 y
a ARVD = arrhythmogenic right ventricular dysplasia; CABG = coronary
artery bypass grafting; ICD = implantable cardioverter-defibrillator;
IDCM = ischemic dilated cardiomyopathy; LVEF = left ventricular ejec-
tion fraction; MI = myocardial infarction; NIDCM = nonischemic dilated
cardiomyopathy; NYHA = New York Heart Association; PTCA = percu-
Data from the Centers for Medicare and Medicaid Services 2005 guidelines.




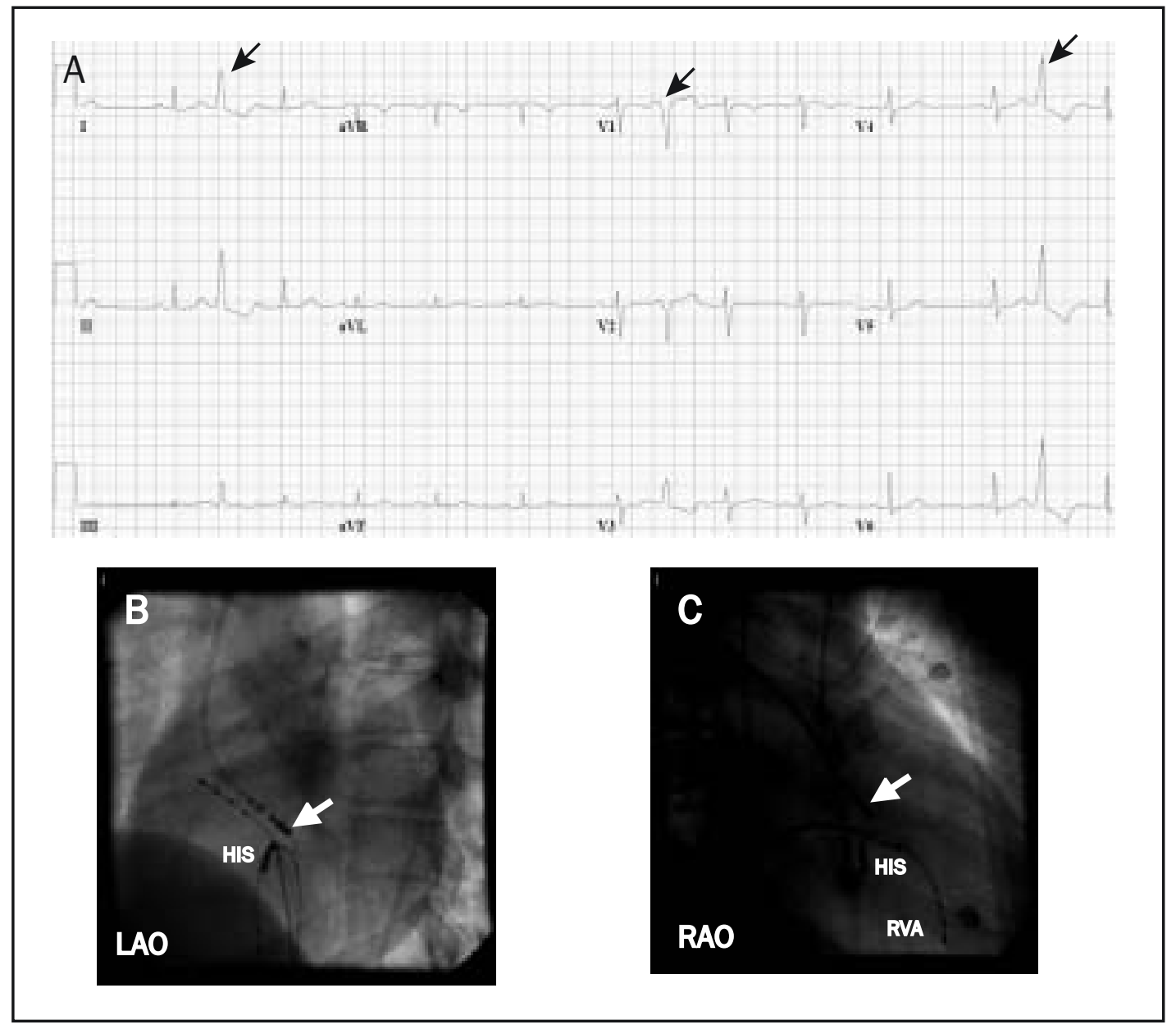

FIGURE 5. Electrograms showing premature ventricular contractions originating from the aortic annulus. This patient had severely symptomatic premature ventricular contractions and nonsustained ventricular tachycardia of the same morphology. A, The premature ventricular contractions (arrows) have a left bundle branch block configuration, indicating an origin in the right ventricle or septal aspect of the left ventricle. The frontal plane axis is directed inferiorly (dominant R wave in leads II and III). This morphology is consistent with an origin in the outflow region of the left or right ventricle. $\mathrm{B}$ and $\mathrm{C}$, Successful site of ablation in the aortic annulus on fluoroscopy. The focus was found at the aortic annulus above the His bundle region (HIS) (white arrow at ablation catheter that enters the ventricle from a retrograde aortic approach). LAO = left anterior oblique; RAO = right anterior oblique; RVA = right ventricular apex.

MI or coronary revascularization or several months after a new diagnosis of nonischemic cardiomyopathy.

\section{Catheter Ablation for VT}

Catheter ablation has the potential to control recurrent VT without the adverse effects of antiarrhythmic drug therapy. As such, it has an important role in nearly all forms of monomorphic VT that are recurrent and not responsive to medical therapy.

Idiopathic VT. In patients with symptomatic idiopathic VT, catheter ablation is a reasonable option when $\beta$-blockers or nondihydropyridine calcium channel blockers fail or are undesirable. For VTs that originate from the outflow tract regions, ablation is successful in $80 \%$ to $90 \%$ of patients.
Figure 5 displays images and electrograms from a patient with an idiopathic outflow tract VT. Failure of ablation is often due to inability to provoke the VT for mapping. Approximately $10 \%$ to $20 \%$ originate outside the RVOT in sleeves of myocardium around the aortic annulus, pulmonary artery, and epicardium and along the mitral annulus. Ablation can be more difficult in these locations and is occasionally prevented by proximity to a coronary artery. Success is achieved in greater than $80 \%$ of patients. ${ }^{47,51,52}$ Complications are infrequent but can include perforation with tamponade and rare coronary occlusions for ablation in the epicardium or aortic root.

It is increasingly recognized that very frequent idiopathic VTs or premature ventricular contractions can cause 


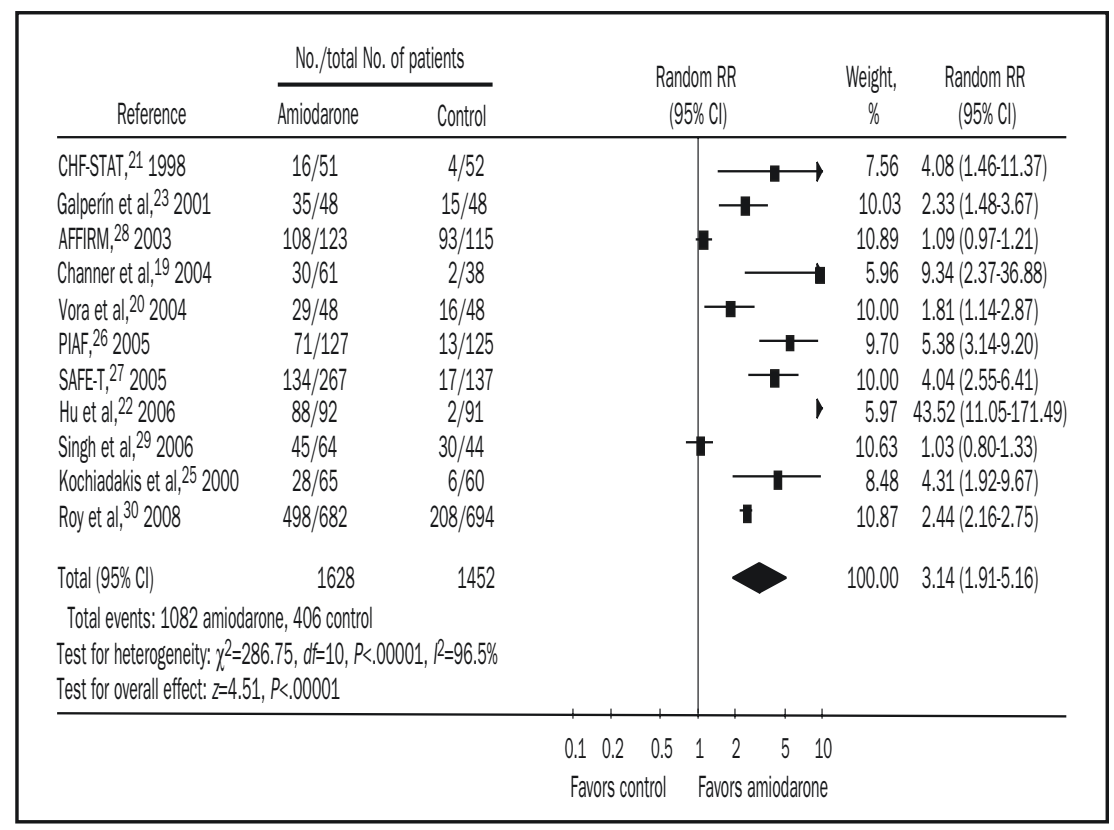

FIGURE 6. Voltage map of the left ventricle from a patient with a prior anterior wall infarction viewed from the left lateral and inferior aspect. Colors represent the bipolar electrogram amplitude. Purple represents normal amplitude $(>1.5 \mathrm{mV})$. Electrogram amplitude is progressively lower as colors progress from blue to green to yellow to red. Gray areas are electrically unexcitable, indicating dense fibrosis. Ventricular tachycardia circuits are located in the scar area. For ablation of ventricular tachycardia, additional mapping is required to define the location of the reentry circuit within the scar.

depressed ventricular function that is a form of tachycardiainduced cardiomyopathy. In some cases, depressed left ventricular function may be an artifact reflecting the difficulty of measuring function in the presence of very frequent arrhythmia. Nonetheless, abolition of the arrhythmia can be followed by marked improvement in ventricular function. ${ }^{53}$

Scar-Related VTs in Structural Heart Disease. In patients with structural heart disease, catheter ablation has a role in preventing or reducing symptomatic scar-related ischemic VTs. ${ }^{54}$ Figure 6 displays a voltage map obtained from a patient with a scar-related VT due to prior MI. Catheter ablation can be life-saving for patients with incessant VT. Success rates for emergent procedures vary from $50 \%$ to $80 \%$, with a rate of major complications of between $2 \%$ and $10 \% .{ }^{55-58}$ Procedure mortality is approximately $3 \%$, with most deaths due to failure of the procedure to control frequent, life-threatening VT. Ablation is also useful for controlling recurrent VT in the setting of dilated cardiomyopathy, infiltrative heart disease such as cardiac sarcoidosis, or ARVD. Ablation in these disorders is often more difficult than ablation for VT due to prior MI. The areas of scar and reentry circuits are more often subepicardial or intramural, requiring epicardial mapping and ablation. Progression of the disease process is likely a factor that increases recurrence of VT during long-term follow-up. ${ }^{59-62}$
Identification of the arrhythmogenic substrate giving rise to VT has been improved by methods that allow identification of scars as regions of low electrogram amplitude in "voltage maps" that can be created by ventricular mapping during stable sinus rhythm. These anatomic reconstructions allow targeting during stable sinus rhythm, while confining ablation lesions to abnormal regions, in the hope of reducing the risk of damage to normal myocardium. This approach, known as substrate mapping, allows ablation to target VTs that are hemodynamically unstable, such that mapping cannot be performed during VT itself.

Failure of VT ablation is often due to a structural problem. Ventricular tachycardia may originate from an epicardial or intramural location. Epicardial scars are particularly common in nonischemic cardiomyopathies but are also seen in some infarct scars and ARVD. A percutaneous subxiphoid pericardial puncture can be used to introduce a mapping and ablation catheter into the pericardial space to target these arrhythmias. This approach is required in approximately $10 \%$ of patients with recurrent VT who are referred for ablation at our center. Care must be taken to avoid ablation near a coronary artery.

In patients with ICDs, the occurrence of an episode of $\mathrm{VT}$ is a marker for increased risk for heart failure and mortality, despite effective VT termination by the ICD. 
Ventricular tachycardia may be a marker for ongoing ventricular remodeling. Whether attempts to prevent or reduce VT in patients with ICDs will improve outcomes is unclear. A recent trial, however, randomized ICD recipients to ablation vs no ablation on the basis of substrate mapping. ${ }^{63}$ During follow-up of 128 patients, ablation reduced spontaneous VT (12\% vs 33\% incidence of spontaneous ventricular arrhythmia requiring ICD therapy; $P=.007)$. Although the trial was not sufficiently powered to assess mortality, there was a favorable trend toward a reduction in mortality in the ablation group.

Polymorphic VT. Recurrent polymorphic VT is less frequent than monomorphic VT. It occurs in patients with Brugada syndrome, those with the long QT syndrome, and in rare patients with idiopathic VF. In rare cases of recurrent polymorphic VT initiated by premature ventricular beats, the premature beats have been effectively targeted for ablation with abolition of recurrent polymorphic VT. Foci are often identified in the ventricular Purkinje system or RVOT.

\section{CONCLUSION}

Ventricular tachycardia is an important cause of sudden death. The risk and consequently the therapeutic approach are determined by the underlying heart disease. Ventricular tachycardia is most commonly associated with ischemic heart disease or other forms of structural heart disease that are associated with a risk of sudden death. Several groups of patients who are at increased risk of sudden death from VT have been identified and benefit from ICDs. It is important to distinguish high-risk groups from patients with idiopathic VT without structural heart disease that has a more benign prognosis.

\section{REFERENCES}

1. Coronel R, Fiolet JW, Wilms-Schopman JG, Opthof T, Schaapherder AF, Janse MJ. Distribution of extracellular potassium and electrophysiologic changes during two-stage coronary ligation in the isolated, perfused canine heart. Circulation. 1989;80(1):165-177.

2. Pogwizd SM, Corr PB. Mechanisms underlying the development of ventricular fibrillation during early myocardial ischemia. Circ Res. 1990; 66(3):672-695.

3. Wit AL, Allessie MA, Bonke FI, Lammers W, Smeets J, Fenoglio JJ Jr. Electrophysiologic mapping to determine the mechanism of experimental ventricular tachycardia initiated by premature impulses: experimental approach and initial results demonstrating reentrant excitation. Am J Cardiol. 1982; 49(1):166-185.

4. El-Sherif N, Smith RA, Evans K. Canine ventricular arrhythmias in the late myocardial infarction period, 8: epicardial mapping of reentrant circuits. Circ Res. 1981;49(1):255-265.

5. Lloyd EA, Zipes DP, Heger JJ, Prystowsky EN. Sustained ventricular tachycardia due to bundle branch reentry. Am Heart J. 1982;104(5, pt 1):10951097.

6. Caceres J, Jazayeri M, McKinnie J, et al. Sustained bundle branch reentry as a mechanism of clinical tachycardia. Circulation. 1989;79(2):256-270.

7. Cohen TJ, Chien WW, Lurie KG, et al. Radiofrequency catheter ablation for treatment of bundle branch reentrant ventricular tachycardia: results and long-term follow-up. J Am Coll Cardiol. 1991;18(7):1767-1773.
8. Tchou P, Jazayeri M, Denker S, Dongas J, Caceres J, Akhtar M. Transcatheter electrical ablation of right bundle branch: a method of treating macroreentrant ventricular tachycardia attributed to bundle branch reentry. Circulation. 1988;78(2):246-257.

9. Splawski I, Shen J, Timothy KW, et al. Spectrum of mutations in longQT syndrome genes: KVLQT1, HERG, SCN5A, KCNE1, and KCNE2. Circulation. 2000;102(10):1178-1185.

10. Brugada $P$, Brugada J. Right bundle branch block, persistent ST segment elevation and sudden cardiac death: a distinct clinical and electrocardiographic syndrome: a multicenter report. J Am Coll Cardiol. 1992;20(6):13911396.

11. Leenhardt A, Lucet V, Denjoy I, Grau F, Ngoc DD, Coumel P. Catecholaminergic polymorphic ventricular tachycardia in children: a 7-year follow-up of 21 patients. Circulation. 1995;91(5):1512-1519.

12. Priori SG, Napolitano $\mathrm{C}$, Tiso $\mathrm{N}$, et al. Mutations in the cardiac ryanodine receptor gene (hRyR2) underlie catecholaminergic polymorphic ventricular tachycardia. Circulation. 2001;103(2):196-200.

13. Postma AV, Denjoy I, Hoorntje TM, et al. Absence of calsequestrin 2 causes severe forms of catecholaminergic polymorphic ventricular tachycardia. Circ Res. 2002;91(8):e21-e26.

14. Gemayel C, Pelliccia A, Thompson PD. Arrhythmogenic right ventricular cardiomyopathy. J Am Coll Cardiol. 2001;38(7):1773-1781.

15. Dalal D, Nasir K, Bomma C, et al. Arrhythmogenic right ventricular dysplasia: a United States experience. Circulation. 2005 Dec 20;112(25):38233832. Epub 2005 Dec 12.

16. Jaoude SA, Leclercq JF, Coumel P. Progressive ECG changes in arrhythmogenic right ventricular disease: evidence for an evolving disease. Eur Heart J. 1996;17(11):1717-1722.

17. Brooks R, Burgess JH. Idiopathic ventricular tachycardia: a review. Medicine (Baltimore). 1988;67(5):271-294

18. Buxton AE, Waxman HL, Marchlinski FE, Simson MB, Cassidy D, Josephson ME. Right ventricular tachycardia: clinical and electrophysiologic characteristics. Circulation. 1983;68(5):917-927.

19. Lerman BB, Belardinelli L, West GA, Berne RM, DiMarco JP. Adenosine-sensitive ventricular tachycardia: evidence suggesting cyclic AMP-mediated triggered activity. Circulation. 1986;74(2):270-280.

20. Lerman BB. Response of nonreentrant catecholamine-mediated ventricular tachycardia to endogenous adenosine and acetylcholine: evidence for myocardial receptor-mediated effects. Circulation. 1993;87(2):382-390.

21. Lerman BB, Stein K, Engelstein ED, et al. Mechanism of repetitive monomorphic ventricular tachycardia. Circulation. 1995;92(3):421-429.

22. Belhassen B, Rotmensch HH, Laniado S. Response of recurrent sustained ventricular tachycardia to verapamil. Brit Heart J. 1981;46(6):679-682.

23. Lemery R, Brugada P, Bella PD, Dugernier T, van den Dool A, Wellens HJ. Nonischemic ventricular tachycardia: clinical course and long-term follow-up in patients without clinically overt heart disease. Circulation. 1989;79(5):990-999.

24. ECC Committee, Subcommittees and Task Forces of the American Heart Association. 2005 American Heart Association Guidelines for Cardiopulmonary Resuscitation and Emergency Cardiovascular Care. Circulation. 2005 Dec 13;112(24)(suppl):IV1-IV203. Epub 2005 Nov 28.

25. Zheng ZJ, Croft JB, Giles WH, Mensah GA. Sudden cardiac death in the United States, 1989 to 1998. Circulation. 2001;104(18):2158-2163.

26. Kannel WB, Wilson PW, D'Agostino RB, Cobb J. Sudden coronary death in women. Am Heart J. 1998;136(2):205-212.

27. Rea TD, Pearce RM, Raghunathan TE, et al. Incidence of out-of-hospital cardiac arrest. Am J Cardiol. 2004;93(12):1455-1460.

28. Solomon SD, Zelenkofske S, McMurray JJ, et al; Valsartan in Acute Myocardial Infarction Trial (VALIANT) Investigators. Sudden death in patients with myocardial infarction and left ventricular dysfunction, heart failure, or both [published correction appears in N Engl J Med. 2005;353(7):744]. N Engl J Med. 2005;352(25):2581-2588.

29. Curtis JP, Sokol SI, Wang Y, et al. The association of left ventricular ejection fraction, mortality, and cause of death in stable outpatients with heart failure. J Am Coll Cardiol. 2003;42(4):736-742.

30. Moss AJ, Zareba W, Hall WJ, et al; Multicenter Automatic Defibrillator Implantation Trial II Investigators. Prophylactic implantation of a defibrillator in patients with myocardial infarction and reduced ejection fraction. $N$ Engl $J$ Med. 2002 Mar 21;346(12):877-883. Epub 2002 Mar 19.

31. Bardy GH, Lee KL, Mark DB, et al; Sudden Cardiac Death in Heart Failure Trial (SCD-HeFT) Investigators. Amiodarone or an implantable cardioverterdefibrillator for congestive heart failure [published correction appears in $N$ Engl J Med. 2005;352(20):2146]. N Engl J Med. 2005;352(3):225-237. 
32. La Rovere MT, Pinna GD, Maestri R, et al. Short-term heart rate variability strongly predicts sudden cardiac death in chronic heart failure patients. Circulation. 2003;107(4):565-570.

33. Chow T, Kereiakes DJ, Bartone C, et al. Prognostic utility of microvolt $\mathrm{T}$-wave alternans in risk stratification of patients with ischemic cardiomyopathy. J Am Coll Cardiol. 2006 May 2;47(9):1820-1827. Epub 2006 Apr 19.

34. Nolan J, Batin PD, Andrews R, et al. Prospective study of heart rate variability and mortality in chronic heart failure: results of the United Kingdom heart failure evaluation and assessment of risk trial (UK-heart). Circulation. 1998;98(15):1510-1516.

35. Bänsch D, Böcker D, Brunn J, Weber M, Breithardt G, Block M. Clusters of ventricular tachycardias signify impaired survival in patients with idiopathic dilated cardiomyopathy and implantable cardioverter defibrillators. J Am Coll Cardiol. 2000;36(2):566-573.

36. Buxton AE, Lee KL, Fisher JD, Josephson ME, Prystowsky EN, Hafley G; Multicenter Unsustained Tachycardia Trial Investigators. A randomized study of the prevention of sudden death in patients with coronary artery disease [published correction appears in N Engl J Med. 2000;342(17):1300]. N Engl J Med. 1999;341(25):1882-1890.

37. Nazarian S, Bluemke DA, Lardo AC. Magnetic resonance assessment of the substrate for inducible ventricular tachycardia in nonischemic cardiomyopathy. Circulation. 2005;112(18):2821-2825.

38. Yusuf S, Peto R, Lewis J, Collins R, Sleight P. Beta blockade during and after myocardial infarction: an overview of the randomized trials. Prog Cardiovasc Dis. 1985;27(5):335-371.

39. Pfeffer MA, Braunwald E, Moyé LA, et al; SAVE Investigators. Effect of captopril on mortality and morbidity in patients with left ventricular dysfunction after myocardial infarction: results of the survival and ventricular enlargement trial. N Engl J Med. 1992;327(10):669-677.

40. SOLVD Investigators. Effect of enalapril on mortality and the development of heart failure in asymptomatic patients with reduced left ventricular ejection fractions [published correction appears in N Engl J Med. 1992; 327(24):1768]. N Engl J Med. 1992;327(10):685-691.

41. The cardiac arrhythmia suppression trial [letter]. $N$ Engl J Med. 1989; 321(25):1754-1756.

42. Cardiac Arrhythmia Suppression Trial (CAST) Investigators. Preliminary report: effect of encainide and flecainide on mortality in a randomized trial of arrhythmia suppression after myocardial infarction. N Engl J Med. 1989;321(6): 406-412.

43. Antiarrhythmics versus Implantable Defibrillators (AVID) Investigators. A Comparison of antiarrhythmic-drug therapy with implantable defibrillators in patients resuscitated from near-fatal ventricular arrhythmias. $N$ Engl J Med. 1997;337(22):1576-1583.

44. Kuhlkamp V, Mewis C, Mermi J, Bosch RF, Seipel L. Suppression of sustained ventricular tachyarrhythmias: a comparison of d,l-sotalol with no antiarrhythmic drug treatment. J Am Coll Cardiol. 1999;33(1):46-52.

45. Pratt CM, Waldo AL, Camm AJ. Can antiarrhythmic drugs survive survival trials? Am J Cardiol. 1998;81(6A):24D-34D.

46. Kuck KH, Cappato R, Siebels J, Rüppel R. Randomized comparison of antiarrhythmic drug therapy with implantable defibrillators in patients resuscitated from cardiac arrest: the Cardiac Arrest Study Hamburg (CASH). Circulation. 2000;102(7):748-754.

47. Rodriguez LM, Smeets JL, Timmermans C, Wellens HJ. Predictors for successful ablation of right- and left-sided idiopathic ventricular tachycardia. Am J Cardiol. 1997;79(3):309-314.
48. Bardy GH, Lee KL, Mark DB, et al; Sudden Cardiac Death in Heart Failure Trial (SCD-HeFT) Investigators. Amiodarone or an implantable cardioverter-defibrillator for congestive heart failure. $N$ Engl J Med. 2005; 352(3):225-237.

49. Desai AS, Fang JC, Maisel WH, Baughman KL. Implantable defibrillators for the prevention of mortality in patients with nonischemic cardiomyopathy: a meta-analysis of randomized controlled trials. JAMA. 2004;292(23):2874-2879.

50. Koplan BA, Epstein LM, Albert CM, Stevenson WG. Survival in octogenarians receiving implantable defibrillators. Am Heart J. 2006;152(4):714719.

51. Scheinman MM, Huang S. The 1998 NASPE prospective catheter ablation registry. Pacing Clin Electrophysiol. 2000;23(6):1020-1028.

52. Chinushi M, Aizawa $Y$, Takahashi K, Kitazawa H, Shibata A Radiofrequency catheter ablation for idiopathic right ventricular tachycardia with special reference to morphological variation and long-term outcome. Heart. 1997;78(3):255-261.

53. Bogun F, Crawford $T$, Reich $S$, et al. Radiofrequency ablation of frequent, idiopathic premature ventricular complexes: comparison with a control group without intervention. Heart Rhythm. 2007 Jul;4(7):863-867. Epub 2007 Mar 12

54 Stevenson WG, Soejima K. Catheter ablation for ventricular tachycardia. Circulation. 2007;115(21):2750-2760.

55. Calkins H, Epstein A, Packer D, et al; Cooled RF Multi Center Investigators Group. Catheter ablation of ventricular tachycardia in patients with structural heart disease using cooled radiofrequency energy: results of a prospective multicenter study. J Am Coll Cardiol. 2000;35(7):1905-1914.

56. Reddy VY, Neuzil P, Taborsky M, Ruskin JN. Short-term results of substrate mapping and radiofrequency ablation of ischemic ventricular tachycardia using a saline-irrigated catheter. J Am Coll Cardiol. 2003;41(12):22282236.

57. Soejima K, Suzuki M, Maisel WH, et al. Catheter ablation in patients with multiple and unstable ventricular tachycardias after myocardial infarction: short ablation lines guided by reentry circuit isthmuses and sinus rhythm mapping. Circulation. 2001;104(6):664-669.

58. Wilber DJ, Kopp DE, Glascock DN, Kinder CA, Kall JG. Catheter ablation of the mitral isthmus for ventricular tachycardia associated with inferior infarction. Circulation. 1995;92(12):3481-3489.

59. Soejima K, Stevenson WG, Sapp JL, Selwyn AP, Couper G, Epstein LM. Endocardial and epicardial radiofrequency ablation of ventricular tachycardia associated with dilated cardiomyopathy: the importance of low-voltage scars. $J$ Am Coll Cardiol. 2004;43(10):1834-1842.

60. Koplan BA, Soejima K, Baughman K, Epstein LM, Stevenson WG. Refractory ventricular tachycardia secondary to cardiac sarcoid: electrophysiologic characteristics, mapping, and ablation. Heart Rhythm. 2006 Aug;3(8): 924-929. Epub 2006 Mar 30.

61. Zeppenfeld K, Schalij MJ, Bartelings MM, et al. Catheter ablation of ventricular tachycardia after repair of congenital heart disease: electroanatomic identification of the critical right ventricular isthmus. Circulation. $2007 \mathrm{Nov}$ 13;116(20):2241-2252. Epub 2007 Oct 29.

62. Dalal D, Jain R, Tandri H, et al. Long-term efficacy of catheter ablation of ventricular tachycardia in patients with arrhythmogenic right ventricular dysplasia/cardiomyopathy. J Am Coll Cardiol. 2007 Jul 31;50(5):432-440. Epub 2007 Jul 16.

63. Reddy VY, Reynolds MR, Neuzil P, et al. Prophylactic catheter ablation for the prevention of defibrillator therapy. N Engl J Med. 2007;357(26):26572665

\section{The Symposium on Cardiovascular Diseases will continue in the April issue.}

